\title{
ARTERITIS DE TAKAYASU REPORTE DE 2 CASOS
}

\author{
John Liévano MD*, Luis Fernando Muñoz Acosta MD**
}

\section{Resumen}

Las vasculitis son enfermedades infrecuentes y aún más aquellas que comprometen las arterias de grande y de mediano calibre, como la de Takayasu y la arteritis temporal. Se presenta el caso de una paciente joven que cumplió los criterios del Colegio Americano de Reumatología para arteritis de Takayasu, a quien se le realizó angiografía para evaluar la extensión de la enfermedad y el enfoque terapéutico. El segundo caso con diagnóstico previo de arteritis de Takayasu corresponde a una mujer menor de 40 años con diferentes compromisos vasculares a quien se le practicó angiografía para evaluar nuevos compromisos arteriales por claudicación de miembros superiores y observar el estado de los stents implantados con anterioridad en la aorta torácica descendente, la carótida común izquierda y las arterias renales.

Palabras clave: arteritis de Takayasu, vasculitis, arteritis.

\section{TAKAYASU ARTERITIS A REPORT OF TWO CASES}

\begin{abstract}
Vasculitis is uncommon, and large or median vessel vasculitis, such as Takayasu and temporal arteritis, are even more rare forms of this disorder. The first case corresponds to a young woman who met the American College of Rheumatology criteria for Takayasu arteritis. The extension of arterial involvement and treatment options were assessed by angiography. The second case herein presented is a less than $\mathbf{4 0}$ year old woman previously diagnosed with Takayasu arteritis with various forms of vascular involvement. She underwent an angiography to identify new arterial involvement for presenting with intermittent claudication of the upper limbs and to evaluate patency of previously implanted descending thoracic aorta, left common carotid artery and renal arteries stents.
\end{abstract}

Key words: Takayasu arteritis, vasculitis, arteritis.

Fecha recibido: febrero II de 2013 - Fecha aceptado: septiembre 4 de 2013

* Profesor Titular, Fundación Universitaria de Ciencias de la Salud. Cardiólogo intervencionista de los hospitales de San José, San Rafael y Saludcoop de la 104. Bogotá DC, Colombia.
** Residente II de Cardiología, Fundación Universitaria de Ciencias de la Salud. Bogotá DC, Colombia. 


\section{Introducción}

La clasificación de arteritis de Takayasu por el ACR (American College of Rheumatology) implica el cumplimiento de tres criterios a partir de los seis propuestos por ellos: edad inferior a 40 años, claudicación de las extremidades en especial las superiores, disminución del pulso braquial, diferencia de la presión arterial sistólica en más $10 \mathrm{~mm} \mathrm{Hg}$ entre ambos miembros superiores, soplo sobre la aorta o la arteria subclavia y anomalías en el estudio angiográfico, siendo este último un criterio más relevante (Tabla 1). La hipertensión se incluyó como criterio, ya que puede ser el síntoma presente en niños y jóvenes por compromiso de arterias renales. La arteritis de Takayasu es una enfermedad inflamatoria de causa desconocida que está clasificada dentro de las vasculitis autoinmunes, que afecta a los grandes vasos cardíacos, sobre todo la aorta y sus ramas principales, aunque también puede comprometer las arterias pulmonares y las coronarias. Los casos que se describen a continuación presentan tres o más de los criterios clínicos y paraclínicos descritos por el ACR.

\section{Caso 1}

Mujer de 35 años con cuadro de varios años de evolución de astenia, adinamia, disnea II/IV y claudicación de miembros superiores, encontrando al examen físico hipertensión arterial de predominio en miembros inferiores, ausencia de pulso de miembro superior izquierdo y alteraciones neurológicas definidas como cefalea y vértigo. En una valoración previa le solicitaron una angiotomografia computarizada de tórax que reporta irregularidad intimal difusa de la aorta con engrosamiento circunferencial sugestivo de trombo mural. El doppler carotídeo revela trombosis con recanalización parcial a nivel de la carótida común izquierda y estenosis de un 50 a $70 \%$ de la carótida interna ipsilateral. El doppler de las arterias vertebrales es normal. Es referida para aortograma toracoabdominal y arteriografía renal, con los siguientes hallazgos:

1. Oclusión total de la arteria carótida común izquierda en su tercio proximal.
2. Oclusión total de la subclavia izquierda en su tercio proximal (Figura 1).

3. Arteriografía renal selectiva bilateral normal.

4. Oclusión total de la rama hepática del tronco celíaco y de la mesentérica superior.

5. Oclusión total de la mesentérica superior en su origen (Figura 2).

6. Mesentérica inferior que da flujo a la mesentérica superior por medio de la arteria cólica izquierda (Figura 3).

7. Hipertensión severa presión sistólica 232, presión diastólica 91, con presión arterial media de 130 $\mathrm{mmHg}$, medidas mediante arteriografía aórtica.

8. Origen y recorrido normal de los vasos coronarios.

\section{Caso 2}

Mujer de 32 años con cuadro de astenia, adinamia y claudicación de miembro superior izquierdo de tres años de evolución. Antecedentes de enfermedad de Takayasu en tratamiento con azatioprina, prednisolona, hipertensión arterial medicada con enalapril $20 \mathrm{mg}$ cada 12 horas, angioplastia e implante de dos stents en arterias renales hace dos años, uno en carótida común izquierda y otro en aorta torácica hace un año. Se realiza estudio angiográfico de aorta toracoabdominal con los siguientes hallazgos:

1. Oclusión total de la arteria subclavia izquierda.

2. Stent en carótida común izquierda permeable (Figura 4).

3. Stent permeable en aorta torácica descendente (Figura 5).

4. Arteria polar superior derecha con stent proximal permeable (Figura 6).

5. Arteria renal izquierda con stent proximal con reestenosis del 50\% (Figura 7).

6. Arteriografía pulmonar selectiva en ambas arterias pulmonares que muestra árbol pulmonar de características agiográficas normales. 


\section{Tabla I. Clasificación del Colegio Americano} de Reumatología*

I. Edad de inicio de la enfermedad menor de 40 años.

2. Claudicación de extremidades, desarrollo y empeoramiento de la fatiga y el malestar de los músculos de una o más extremidades, en especial superiores.

3. Disminución de pulsos de las arterias braquiales.

4. Diferencia de más de $10 \mathrm{~mm} \mathrm{Hg}$ de la presión sistólica entre los brazos.

5. Soplo en arterias subclavias o en aorta abdominal.

6. Arteriografía anormal. Estrechamiento u oclusión de la aorta en su totalidad, o de arterias de las extremidades proximales superiores o inferiores, no debidas a ateroesclerosis ni a displasia fibromuscular.

*Requiere mínimo tres criterios. Sensibilidad 95\%. Especificidad 97.8\%.

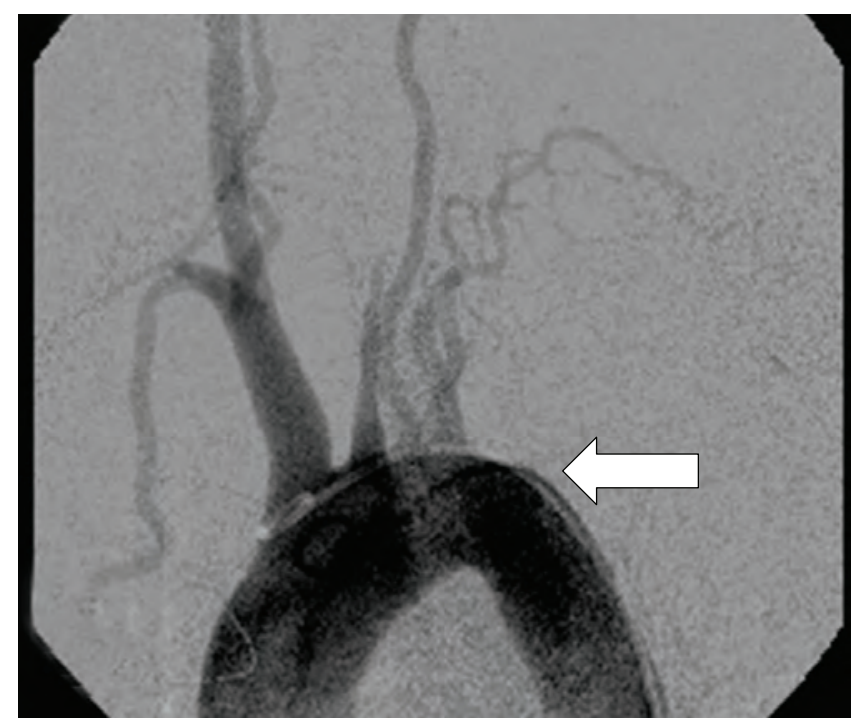

Figura I. Oclusión total de la subclavia izquierda (caso I).

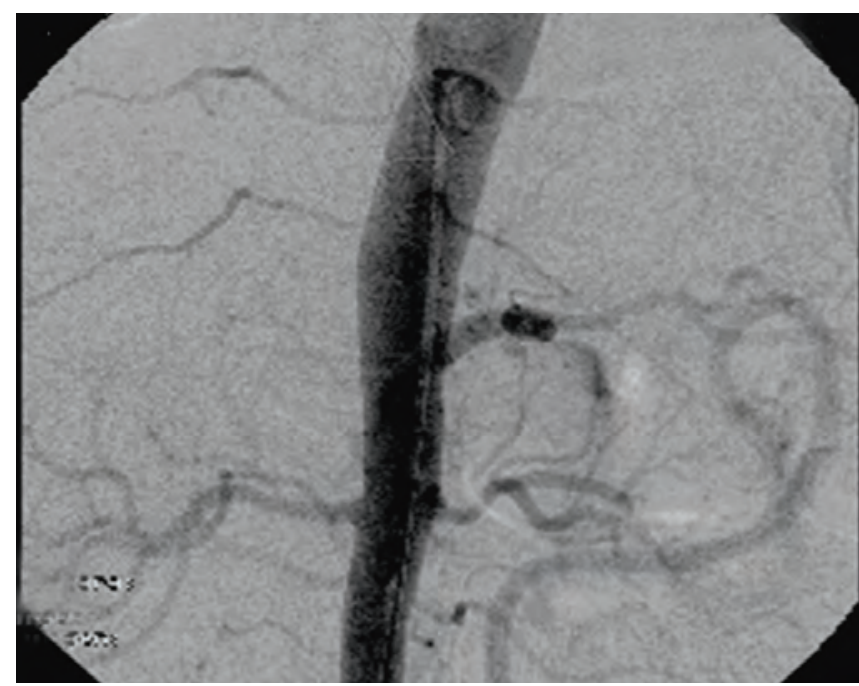

Figura 2. Oclusión total de la arteria mesentérica superior en su origen (caso I).

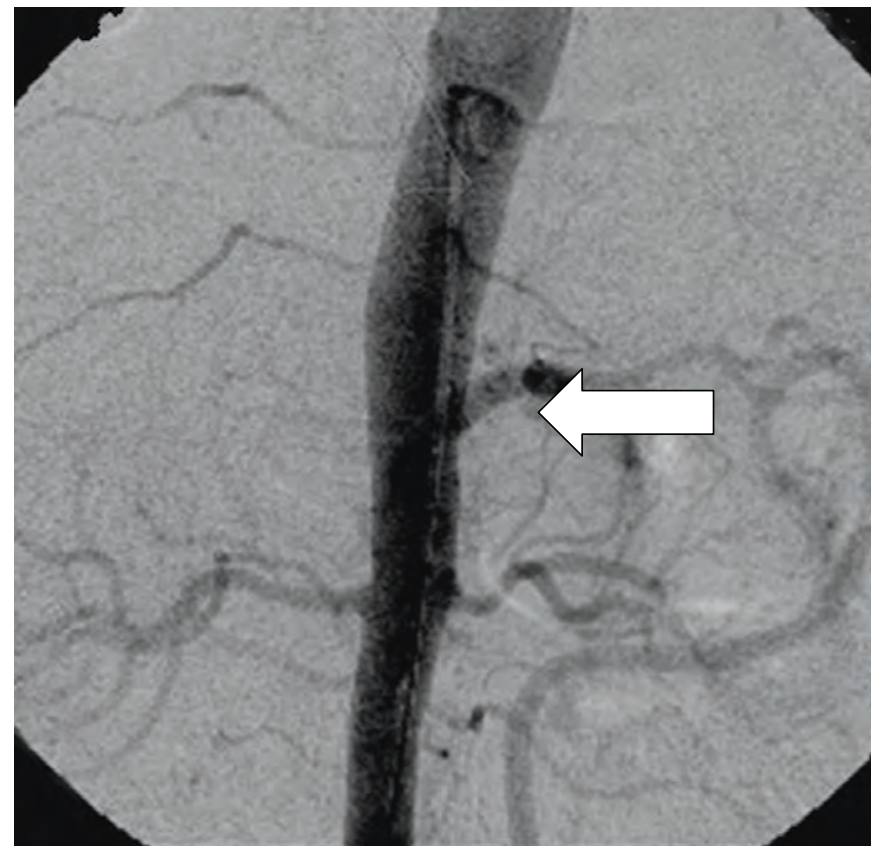

Figura 3. Arteria mesentérica inferior que da flujo a la mesentérica superior por medio de la arteria cólica izquierda (caso I).

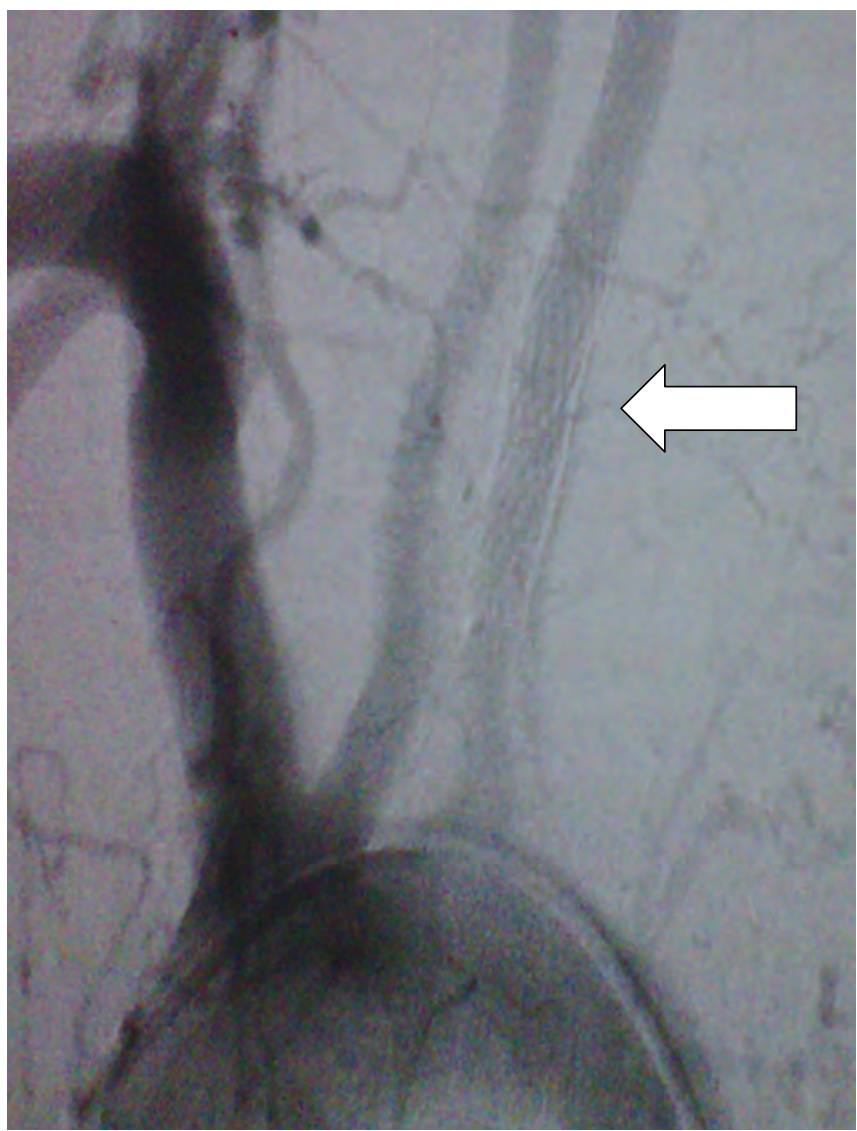

Figura 4. Stent en la arteria carótida común izquierda permeable (caso 2). 




Figura 5. Aorta descendente con stent permeable (caso 2).

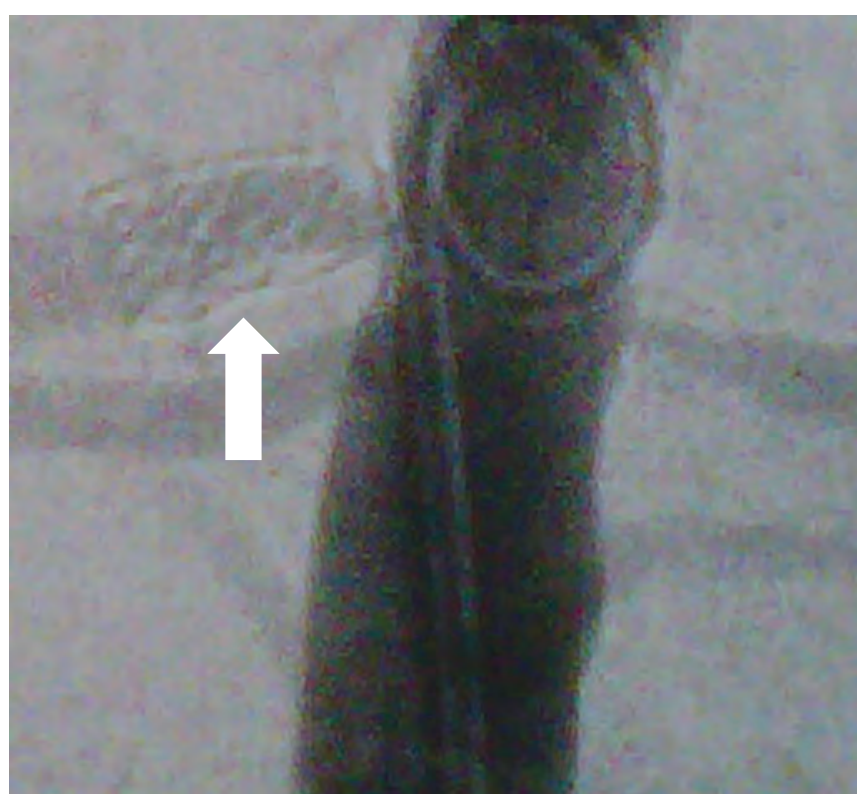

Figura 6. Arteria polar superior derecha con stent permeable (caso 2).

\section{Disc usión}

Al inicio de la atención encontramos que ingresan para realizarles angiografía de aorta y sus ramas dos mujeres en edad reproductiva, menores de 40 años,

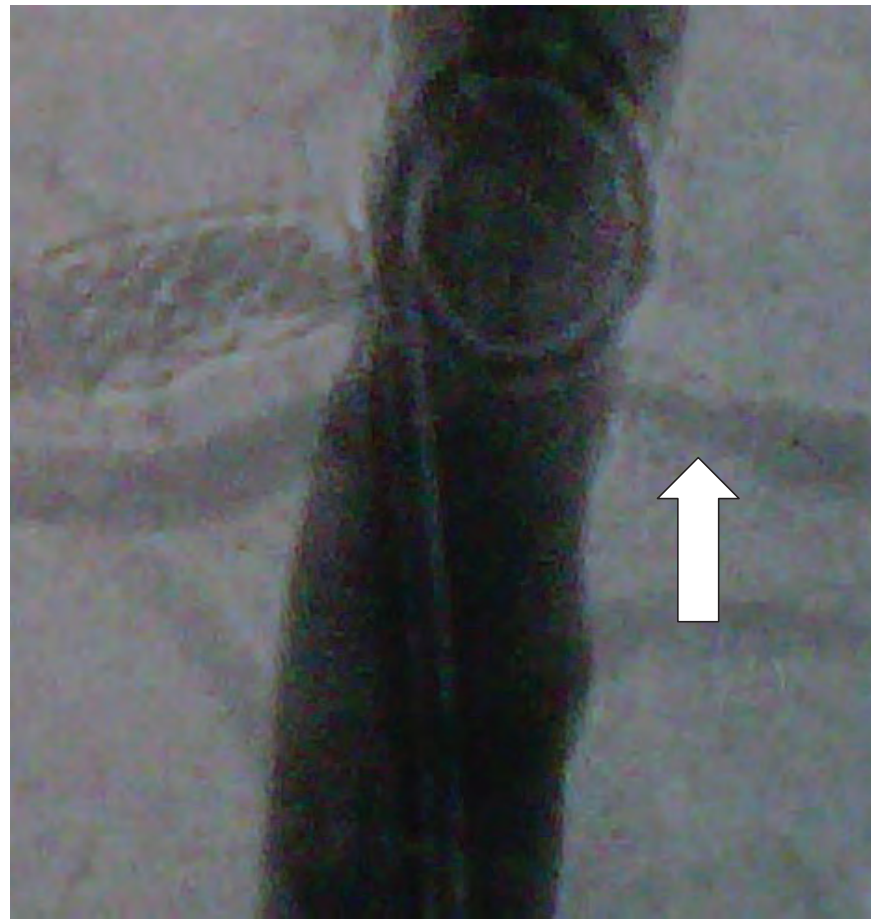

Figura 7. Arteria renal izquierda con stent proximal con reestenosis focal del $50 \%$ (caso 2 ).

quienes coincidían en la sintomatología inicial aunque en épocas diferentes de comienzo. Cursaron con astenia, adinamia, malestar general y al progresar los síntomas presentaron claudicación de miembros superiores con compromiso del pulso del miembro superior izquierdo. Compartían además similitudes en el compromiso de las arterias carótida y subclavia izquierdas (evaluada por cateterismo), hipertensión y diferentes presiones en los miembros superiores. Las diferencias en la presentación de la arteritis de Takayasu en estas dos mujeres en edad fértil radicaba en el compromiso en el caso uno de las arterias hepática y mesentérica superior, sin compromiso de las renales ni de la aorta descendente como se evidencia en el caso dos.

La arteriografía se clasificó según Numan y col. 1997 en categorías de I a V, con subcategorías dependiendo si tienen o no lesiones sobre arterias coronarias y pulmonares. Correspondieron a la categoría V que involucra la aorta ascendente, el arco y sus ramas, la torácica descendente y la aorta abdominal, con y sin compromiso de las arterias renales, evidenciando una gran extensión vascular. ${ }^{1-5}$ 
La arteritis de Takayasu es un proceso inflamatorio que afecta la aorta y sus ramas principales incluidas las arterias coronarias y pulmonares. Es una vasculitis granulomatosa sistémica idiopática de grandes y medianas arterias, conocida también como síndrome del arco aórtico o enfermedad sin pulsos. La incidencia varía según la raza, origen étnico y área geográfica. En Japón es mayor en las mujeres y se estima en 1,2 a 2,6 casos por millón ${ }^{1-5}$ y una de cada 3.000 autopsias tiene características de arteritis de Takayasu. ${ }^{2}$ En general es diez veces más frecuente en mujeres, la edad media de aparición es 25 años, la incidencia en Estados Unidos se estima en 2,6 por un millón de personas y de 1,26 en el norte de Europa.

En Colombia el registro que presentó la Sociedad Colombiana de Reumatología en diciembre de 2006, reporta 540 casos de vasculitis primarias en 62 años, desde 1945 hasta 2006, de los cuales 76 atribuidos a arteritis de Takayasu. Se encontró un incremento significativo en los alelos HLA DRB1 1602, HLA DRB1 1010, a diferencia de otros grupos en el mundo que se encuentra más asociación con HLA DRB1 1602, HLA B52, estos últimos más relacionados con compromiso pulmonar y recaídas frecuentes.

Es de nuestro interés reportar estos casos por la baja frecuencia de presentación y severidad de las lesiones anotando que no solo el manejo farmacológico sino también el endovascular mejoran las obstrucciones arteriales. En el registro de los últimos diez años (2002 al 2012) sobre un total de 22.000 cateterismos en tres instituciones de Bogotá, solo hemos encontrado estas dos pacientes con arteritis de Takayasu.

La causa es desconocida pero la inmunidad celular está implicada en las lesiones vasculares, encontrando una activación de los linfocitos $\mathrm{T}$ acelerada por factores que actúan sobre el sistema inmune, como las infecciones virales..$^{1,2,4,5}$

Las células que predominan son linfocitos T gammadelta citotóxicos y los natural killers. Existen linfocitos B en menor cantidad, citoquinas (IL 6, TNF) y se ha encontrado la proteína del choque térmico de micobacterias (HSP-65) que aún no se ha definido su papel en la génesis de la arteritis. Las características clínicas más relevantes son inflamación sistémica, dolor ocasionando por la vasculitis y signos y síntomas debidos a la estenosis, oclusión o dilatación de los vasos sanguíneos en forma de aneurismas. ${ }^{1-4,6,7}$

Los signos y síntomas asociados con la arteritis de Takayasu son variados y entre los más frecuentes están fiebre de origen desconocido y dolor en cuello que son inespecíficos; después aparecen los que están en relación con lesiones vasculares, causando alteraciones neurológicas, visuales, diferencias de presión arterial entre miembros superiores y falta de pulsos en cualquiera de las extremidades con claudicación, siendo más comunes las de los miembros superiores.

La hipertensión por isquemia renal secundaria a estenosis de una o las dos arterias renales, la coartación de aorta, los infartos pulmonares y la angina de pecho por compromiso de coronarias de predominio ostial son de aparición variable, se han reportado casos de mujeres jóvenes debutando con infarto o muerte. ${ }^{1,8-10}$ La presencia de aneurismas o disecciones aorticas, y la insuficiencia de la válvula aortica pueden llevar a insuficiencia cardiaca. ${ }^{10-12}$

No hay datos típicos hematológicos o bioquímicos que determinen el diagnóstico de arteritis de Takayasu, pero la actividad de la enfermedad se puede evaluar con las cifras de proteína $\mathrm{C}$ reactiva, sedimentación globular, niveles de leucocitos y anemia, factores que favorecen la trombosis, es decir actividad de agregación plaquetaria, fibrinógeno, tiempo de protrombina, tiempo de tromboplastina parcial activado y la antitrombina. Es posible encontrar elevación de inmunoglobulinas $\mathrm{G}$ y $\mathrm{A}, \mathrm{y}$ factores del complemento $\mathrm{C}_{3} \mathrm{y}_{4}$, asociación de aumento de antígeno leucocitario (HLA)-B52 donde parece coincidir con lesiones más graves que los asociados con HLA-B39.

La presencia o no de estenosis o dilataciones arteriales se pueden evaluar con angiografía por sustracción digital (DSA), tomografía computarizada tridimensional (CT-3D) y F-18-fluorodeoxiglucosa por emisión de positrones (PET-FDG), que también puede ser útil en el diagnóstico precoz de la enfermedad, pues nos 
permite ver la pared del vaso y su actividad metabólica implicada en el proceso inflamatorio. ${ }^{1,3,6} \mathrm{La} \mathrm{RM}$ aporta datos adicionales por valorar la pared vascular como primera manifestación de la enfermedad, al igual que para evaluar la extensión y el seguimiento., 2,13

Una vez efectuado el diagnóstico de arteritis de Takayasu se debe iniciar manejo con glucocorticoides como terapia de base, la mayor parte de los pacientes responden en forma adecuada. Cuando hay respuesta clínica y paraclínica se debe disminuir después de dos semanas hasta una dosis de mantenimiento de 5 a 10 $\mathrm{mg} /$ día, para retirarlos siempre que sea posible.

Aunque algunos pacientes responden bien a los esteroides, hay otros que presentan recaídas frecuentes, para lo cual se usan medicinas inmunosupresoras como metotrexate, azatioprina, micofenolatomofetilo, rituximab, infliximab, ciclosporina, plasmaferisis, ciclofosfamida o procedimientos de revascularización endovascular o quirúrgica. ${ }^{8,14}$

Algunas publicaciones reportan el uso de gammaglobulina endovenosa para los pacientes con recaídas con esteroides o a la combinación de estos con otros medicamentos, sabiendo que el fundamento del manejo con inmunoglobulina es la modulación de la respuesta inmune que es alentadora en muchos casos. ${ }^{8,14}$

La presentación y discusión anterior nos permite ver que debemos esforzarnos por entender algunas enfermedades infrecuentes con respecto a su forma de inicio, progresión y estado avanzado, así como de comprender las posibles opciones terapéuticas, pero por el hecho de ser enfermedades infrecuentes no son ajenas a nuestra realidad. Para finalizar es necesario sensibilizarnos con la arteritis de Takayasu y demás vasculitis y así realizar un diagnóstico temprano.

\section{Referencias}

1. JCS Joint Working Group. Guideline for management of vasculitis syndrome (JCS 2008). Japanese Circulation Society. Circ J. 2011; 75(2):474-503.

2. Dufrechou C, Cedrés S, Robaina R, Bagattini JC. Arteritis de Takayasu: revisión de criterios diagnósticos y terapéuticos a propósito de un caso clínico. Rev. Méd. Urug. 2006; 22(3):236-40.

3. Mandell BF, Hoffman, Gary S. Forms of vasculitis relevant to cardiologists and cardiovascular surgeons Takayasu arteritis. In: Bonow RO, Mann DL, Zipes DP, Libby P. Braunwald's heart disease. Philadelphia : Elsevier Saunders; 2004.

4. Le T, Baudendistel T, Chin-Hong P, Lai C. First aid for the internal medicine boards. New York: McGraw-Hill Medical; 2011.

5. Harrison TR, Kasper DL. Harrison : principios de medicina interna. México: McGraw-Hill; 2006.

6. Jayne D. The diagnosis of vasculitis. Best Pract Res Clin Rheumatol. 2009 Jun; 23(3): 445-53.

7. Andrews J, Mason JC. Takayasu's arteritis--recent advances in imaging offer promise. Rheumatology (Oxford). 2007;46(1):6-15.

8. Tanaka F, Kawakami A, Iwanaga N, Tamai M, Izumi Y, Aratake K, et al. Infliximab is effective for Takayasu arteritis refractory to glucocorticoid and methotrexate. Intern Med. 2006;45(5):313-6.

9. Matsuura K, Ogino H, Kobayashi J, Ishibashi-Ueda H, Matsuda H, Minatoya K, et al. Surgical treatment of aortic regurgitation due to Takayasu arteritis: longterm morbidity and mortality. Circulation. 2005;112(24):3707-12.

10. Ouali S, Kacem S, Ben Fradj F, Gribaa R, Naffeti E, Remedi F, et al. Takayasu arteritis with coronary aneurysms causing acute myocardial infarction in a young man. Tex Heart Inst J. 2011; 38(2):183-6.

11. Aguilera B. Arteritis de Takayasu de la coronaria izquierda: presentación de un caso que debuta con muerte súbita. Cuad. med. forense. 2006; 45-46:184-5.

12. Aguirre M, Restrepo CA. Arteritis de Takayasu en una joven de 15 años. Acta Med Colomb. 2005;30(4):281-4.

13. Yague-Romeo D, Angulo-Hervías E, Hueso-del Rio LE. Utilidad de la tomografia computarizada y de la resonancia magnética en el diagnóstico de la arteritis de takayasu. Rev Clin Esp. 2010;210(4):e11-12.

14. Velásquez CJ, Brome AP, Benjumea LM, Cardona DC, Tobón EA, Orrego LD, et al. Respuesta a gamaglobulina endovenosa en arteritis de Takayasu de difícil manejo. Acta Med Colomb. 2010;35(3):139-42. 